\title{
Pseudo-scalar mesons at finite temperatures from a Dyson-Schwinger-Bethe-Salpeter approach
}

\author{
Sergey Dorkin ${ }^{1,2 *}$, Leonid Kaptari $^{1 * *}$, and Burkhard Kämpfer $^{3}$ \\ ${ }^{1}$ Bogoliubov Laboratory of Theoretical Physics, 141980, JINR, Dubna, Russia \\ ${ }^{2}$ International University Dubna, Dubna, Russia \\ ${ }^{3}$ Helmholtz-Zentrum Dresden-Rossendorf, PF 510119, 01314 Dresden, Germany
}

\begin{abstract}
The truncated Dyson-Schwinger-Bethe-Salpeter equations are employed at non-zero temperature. The truncations refer to a rainbow-ladder approximation augmented with an interaction kernel which facilitates a special temperature dependence. At low temperatures, $T \rightarrow 0$, we recover a quark propagator from the Dyson-Schwinger (gap) equation smoothly interpolating to the $T=0$ results. Utilizing that quark propagator we evaluate the BetheSalpeter vertex function in the pseudo-scalar $q \bar{q}$ channel for the lowest boson Matsubara frequencies and find a competition of $q \bar{q}$ bound states and quasi-free two-quark states at $T=O(100 \mathrm{MeV})$.
\end{abstract}

\section{Introduction}

The description of mesons as quark-antiquark bound states within the framework of the Bethe-Salpeter (BS) equation with momentum dependent quark mass functions, determined by the Dyson-Schwinger (DS) equation, is able to explain successfully many spectroscopic data, such as meson masses, electromagnetic properties of pseudoscalar mesons and their radial excitations, and other observables [1-13]. Such a formalism maintains important features of QCD, e.g. dynamical chiral symmetry breaking, dynamical quark dressing, requirements of the renormalization group theory etc., cf. [14]. The main ingredients here are the full quark-gluon vertex function and the dressed gluon propagator, which are entirely determined by the running coupling and the bare quark mass parameters. The approach is based on an effective model, known as the rainbow-ladder approximation [4], which specifies the dressed vertex function $\Gamma_{\mu} \rightarrow \gamma_{\mu}$ (for quark-gluon coupling) and interaction kernel $g^{2} D_{\mu \nu}(k) \rightarrow \mathcal{G}\left(k^{2}\right) D_{\mu \nu}^{f r e e}(k)$ within the Landau gauge for the gluon propagator. Here, $\gamma_{\mu}$ is a Dirac gamma matrix and $D_{\mu \nu}$ stands for the gluon propagator; $g$ is the coupling strength and $k$ denotes a momentum. At finite temperatures, the approach is generalized within the imaginary-time formalism, which uses the discrete Matsubara frequencies [15-17]. Consequently, the interaction kernel and the DS and BS solutions become also discrete with respect to these frequencies.

The hot environment may modify the hadron masses, life times (decay constants) etc. and, at sufficiently large temperature, in hot and dense strongly interacting matter, (phase)

\footnotetext{
*e-mail: dorkin@theor.jinr.ru

**e-mail: kaptari@theor.jinr.ru
} 
transitions may occur, related to quark deconfinement phenomena, as e.g. dissociation of hadrons into quark-gluon degrees of freedom.

We calculate the pole masses of pseudo-scalar quark-antiquark bound-states for the few first Matsubara frequencies and investigate their dependence on temperature in a large interval, including the "dissociation region". We anticipate that the main peculiarities of masses computed at some particular Matsubara frequencies will reflect the general $T$ dependence of the inertial masses at high temperatures. We treat the bound states within the BS formalism within the same approach as the one used in solving the DS equation, i.e. with the rainbow truncation and Alkofer-Watson-Weigel (AWW) interaction kernel [12].

\section{Basic Formulae}

\subsection{Truncated Dyson-Schwinger and Bethe-Salpeter equations in vacuum}

To determine the bound-state mass of a quark-antiquark pair one needs to solve the DS and the homogeneous BS equations, which in the rainbow ladder approximation and in Euclidean space read

$$
\begin{aligned}
& S^{-1}(p)=S_{0}^{-1}(p)+\frac{4}{3} \int \frac{d^{4} k}{(2 \pi)^{4}}\left[g^{2} D_{\mu \nu}(p-k)\right] \gamma_{\mu} S(k) \gamma_{v}, \\
& \left.\Gamma(P, p)=-\frac{4}{3} \int \frac{d^{4} k}{(2 \pi)^{4}} \gamma_{\mu} S\left(\eta_{1} P+k\right) \Gamma(P, k) S\left(-\eta_{2} P+k\right)\right) \gamma_{v}\left[g^{2} D_{\mu \nu}(p-k)\right],
\end{aligned}
$$

where $\eta_{1}$ and $\eta_{2}=1-\eta_{1}$ are the partitioning parameters defining the quark momenta as $p_{1,2}=k \pm \eta_{1,2} P$ with $P, p$ and $k$ denoting the total and relative momenta of the bound system, respectively ${ }^{1} ; \Gamma(P, k)$ stands for the tBS vertex function being a $4 \times 4$ matrix, $S_{0}(p)=(i \gamma \cdot p+m)^{-1}$ and $S(p)=(i \gamma \cdot p A(p)+B(p))^{-1}$ are the propagators of bare and dressed quarks, respectively, with mass parameter $m$ and the dressing functions $A(p)$ and $B(p)$. At zero temperature the above equations are $\mathrm{O}(4)$ invariant and the propagator functions $A(p)$ and $B(p)$ depend solely on $p^{2}=\mathbf{p}^{2}+p_{4}^{2}$. The total momentum $P=\left(\mathbf{0}, i M_{q \bar{q}}\right)$, for a particle at rest, is an external parameter for (2); the momenta of individual quarks squared are $p_{1,2}^{2}=-M_{q \bar{q}}^{2} / 4+k^{2} \pm i M_{q q} k_{4}$, where the Euclidean components $\mathbf{k}$ and $k_{4}$ of the relative momentum are defined as $k^{2}=\mathbf{k}^{2}+k_{4}^{2}$ which, for the BS equation, are the integration variables.

Following examples in the literature $[4,7,8,12]$ the interaction kernel in the rainbow approximation in the Landau gauge is chosen as

$$
\begin{aligned}
& g^{2}\left(k^{2}\right) \mathcal{D}_{\mu v}\left(k^{2}\right)=\left(D_{I R}\left(k^{2}\right)+D_{U V}\left(k^{2}\right)\right)\left(\delta_{\mu \nu}-\frac{k_{\mu} k_{v}}{k^{2}}\right) \\
& D_{I R}\left(k^{2}\right)=\frac{4 \pi^{2} D k^{2}}{\omega^{6}} e^{-k^{2} / \omega^{2}}, \quad D_{U V}\left(k^{2}\right)=\frac{8 \pi^{2} \gamma_{m} F\left(k^{2}\right)}{\ln \left[\tau+\left(1+\frac{k^{2}}{\Lambda_{Q C D}^{2}}\right)^{2}\right]},
\end{aligned}
$$

where the first term originates from the effective infrared (IR) part of the interaction determined by soft, nonperturbative effects, while the second one ensures the correct ultraviolet (UV) asymptotic behaviour of the QCD running coupling, $F\left(k^{2}\right)=\left\{1-\exp \left(-k^{2} /\left[4 m_{t}^{2}\right]\right)\right\} / k^{2}$ with $m_{t}$ as an adjustable parameter, $m_{t} \simeq 0.5 \mathrm{GeV}$, and $\tau=e^{2}-1, \Lambda_{Q C D}=0.234 \mathrm{GeV}$, and $\gamma_{m}=12 /\left(33-2 N_{f}\right)$ for $N_{f}$ as active flavors. We restrict ourselves to the simplest version

\footnotetext{
${ }^{1}$ Usually, for quarks of masses $m_{1,2}$ the partitioning parameters are chosen as $\eta_{1,2}=m_{1,2} /\left(m_{1}+m_{2}\right)$. However, in general, the tBS solution is independent of the choice of $\eta_{1,2}$.
} 
of the model, namely with the interaction kernel where the UV term (the effect of which is found [18] to be negligibly small) is ignored at all. This interaction is the Alkofer-WatsonWeigel [12] kernel. A prominent feature of such an interaction is that, with only a few adjustable parameters - $D, \omega$ in the IR term and quark mass parameter $m$ in the bare quark propagator $S_{0}$ - it provides a good description of the pseudoscalar, vector and tensor meson mass spectra at zero temperature. At finite temperatures the interaction AWW kernel is kept the same as in vacuum. Notice also that, even at zero temperatures, the tBS equation becomes rather complicate for numerical solutions since it involves the quark propagator functions in the complex Euclidean space, where they can (actually they do) exhibit pole-like singularities. A rather detailed analysis of solving the tBS equation in vacuum in presence of poles has been reported in [5]. Reiterate that, within the rainbow approximation, the Euclidean $P_{4}=i M_{q \bar{q}}$ is an external parameter in the tBS equation, while $k_{4}$ is an integration variable.

At finite temperatures the $O(4)$ symmetry is broken and, consequently, the dependence of the quark propagator on $\mathbf{p}$ and $p_{4}$ requires a separate treatment. To describe the propagator in this case a third function $C$ is needed, besides the functions $A$ and $B$ introduced above for vacuum.

The inverse quark propagator is now parametrized as

$$
S^{-1}\left(\mathbf{p}, \omega_{n}\right)=i \gamma \mathbf{p} A\left(\mathbf{p}^{2}, \omega_{n}^{2}\right)+i \gamma_{4} p_{4} C\left(\mathbf{p}^{2}, \omega_{n}^{2}\right)+B\left(\mathbf{p}^{2}, \omega_{n}^{2}\right) .
$$

Accordingly, the interaction kernel is decomposed in to a transversal and longitudinal part

$$
\left[g^{2} D_{\mu v}\left(\mathbf{k}, \Omega_{m n}\right)\right]=\mathcal{P}_{\mu \nu}^{T} D^{T}\left(\mathbf{k}, \Omega_{m n}, 0\right)+\mathcal{P}_{\mu \nu}^{L} D^{L}\left(\mathbf{k}, \Omega_{m n}, m_{g}\right),
$$

where $\Omega_{m n}=\omega_{m}-\omega_{n}$ and the gluon Debye screening mass $m_{g}$ is introduced in the longitudinal part of the propagator, where $k^{2}=\mathbf{k}^{2}+\Omega_{m n}^{2}+m_{g}^{2}$ enters. The Debye mass describes perturbatively the screening of chromoelectric fields at large temperatures, therefore it is relevant for the perturbative UV term in the limit of quark-gluon plasma where the light-quark bound states do not longer exist, and the system is to be described by quark and gluon degrees of freedom. As for the non-perturbative pure phenomenological IR term, the Debye mass is neglected in the confinement region, i.e. at temperatures below $T_{c}$.

By using the Feynman rules for finite temperatures, see e.g. [16], it can be shown that the gap equation has the same form as in case of $T=0$, Eq. (1), except that within the Matsubara formalism the integration over $k_{4}$ is replaced by the summation over the corresponding frequencies, formally $\int \frac{d^{4} k}{(2 \pi)^{4}} \longrightarrow T \sum_{n=-\infty}^{\infty} \int \frac{d^{3} k}{(2 \pi)^{3}}$. The explicit form of the system of equations for $A, B$ and $C$ to be solved can be found in $[19,20]$.

\section{3 tBS equation at finite temperatures}

\subsection{Partial decomposition of the BS vertex function}

We focus on the tBS equation for pseudo-scalar states. Prior an analysis of the tBS equation at finite temperature, we briefly recall the calculations at $T=0$. The $O(4)$ symmetric solution of Eq. (2) in Minkowski space for the pseudo-scalar mesons can be written in the form (cf. [12, 21]

$$
\Gamma(P, p)=\left[\mathcal{F}_{1}+(p P) \mathcal{F}_{2} \hat{p}+\mathcal{F}_{3} \hat{P}+\mathcal{F}_{4}(\hat{p} \hat{P}-\hat{P} \hat{p})\right] \gamma_{5},
$$

where the scalar vertex functions $\mathcal{F}_{i}$ are functions of $p^{2}$ and even functions of $(p P)$. In (6) the notation $\hat{p}=p^{\mu} \gamma_{\mu}$ and $\hat{P}=P^{\mu} \gamma_{\mu}$ is adopted. It has been found that the contribution of the last term in Eq. (6), proportional to the tensor matrix $\sigma_{\mu \nu}=i\left[\gamma_{\mu}, \gamma_{v}\right] / 2$, is negligibly small [5] 
and, for the qualitative analysis we are interested in the present paper, can be safely omitted. Then, at $T=0$, there remain only three components in the BS vertex.

To extend the equation to finite temperatures one has to take into account the broken $O(4)$ symmetry which requires separate consideration of space-like and time-like products of four vectors. Consequently, the first three terms in Eq. (6) transform into six components:

$$
\begin{aligned}
\Gamma(P, q) & \equiv \tilde{\Gamma}(P, q) \gamma_{5}= \\
& =\frac{1}{2}\left[A_{1}+A_{2} P_{0} \gamma_{0}-A_{3}(\tilde{q} \tilde{p}) q_{0} \gamma_{0}-A_{4} q_{0} P_{0} \hat{\tilde{q}}+A_{5}(\tilde{q} \tilde{p}) \hat{\tilde{q}}-A_{6} \hat{\tilde{p}}\right] \gamma_{5},
\end{aligned}
$$

where the scalar functions $A_{i}$ depend separately on the time, $\left(P_{0}, q_{0}\right)$, and spatial components, $(|\mathbf{P}|,|\mathbf{q}|)$ of 4 -vectors $P$ and $q$. The unit vectors $\tilde{q}$ and $\tilde{p}$ in (7) are purely spatial, i.e. $\tilde{q}=$ $(0, \mathbf{q} /|\mathbf{q}|)$ and $\tilde{p}=(0, \mathbf{P} /|\mathbf{P}|)$. To pass to Euclidean space, recall that for a meson at rest at $T=0$ the four product $(P q)$ transforms as $\left(P_{0} q_{0}\right)_{M} \rightarrow-\left(P_{4} q_{4}\right)_{E}=-i M_{q \bar{q}} q_{4}$, where $q_{4}$ is the integration variable in the BS equation. Within the rainbow approximation, the interaction kernel does not depend on the total momentum $P$. Therefore, in the tBS equation, $P_{4}$ plays the role of an external parameter which defines the pole of the two-particle Green function, $P_{4}^{2}=$ $-M_{q \bar{q}}^{2}$. If the two-particle system is not at rest then $P_{4}^{2}+\mathbf{P}^{2}=(i E)^{2}+\mathbf{P}^{2}=-M_{q \bar{q}}^{2}$, where $E$ is the total energy of the meson. At finite temperatures the Feynman rules in Euclidean space [16] result in the same formal procedure for the tBS equation as the one used in deriving the tDS equation, i.e. formally, the relative momentum becomes discrete, $q=\left(\mathbf{q}, q_{4}\right) \rightarrow q_{n}=\left(\mathbf{q}, \omega_{n}\right)$ and the integration over $q_{4}$ is replaced by summation over the Matsubara frequencies $\omega_{n}$. The total energy of the meson becomes also discrete, $i E \rightarrow i E_{N}=i \Omega_{N}=2 \pi i N T$, where $\Omega_{N}$ is the Matsubara frequency for bosons, with $N \in \mathbb{Z}$. Then the BS equation in Euclidean space reads

$$
\tilde{\Gamma}\left(P_{N}, p_{n}\right)=\frac{4}{3} T \sum_{m} \int \frac{d^{3} q}{(2 \pi)^{3}} \gamma_{\mu} S^{(+)}(1) \tilde{\Gamma}\left(P_{N}, q_{m}\right) \tilde{S}^{(-)}(2) \gamma_{v} D_{\mu \nu}\left(\kappa_{m n}\right),
$$

where $\tilde{\Gamma}$ is defined by Eq. (7), $\kappa_{m n}=\left(\mathbf{p}-\mathbf{q}, \omega_{n}-\omega_{m}\right)$ and $\tilde{S}^{(-)}(2)=\gamma_{5} S^{(-)}(2) \gamma_{5}$. Correspondingly, the quark propagators $S^{(+)}(1)$ and $\tilde{S}^{(-)}(2)$ are

$$
\begin{aligned}
& S^{(+)}(1)=i \vec{\gamma} \cdot(\mathbf{P} / 2+\mathbf{q}) \sigma_{V}(1)-i \gamma_{4} \cdot\left(P_{4} / 2+q_{4}\right) \sigma_{C}(1)+\sigma_{S}(1), \\
& \tilde{S}^{(-)}(2)=i \vec{\gamma} \cdot(\mathbf{P} / 2-\mathbf{q}) \sigma_{V}(2)+i \gamma_{4} \cdot\left(P_{4} / 2-q_{4}\right) \sigma_{C}(2)+\sigma_{S}(2),
\end{aligned}
$$

where $\sigma_{V, C, S}$ are defined by the solution of the tDS equation at the same temperature $T$,

$$
\sigma_{V,(C, S)}(1,2)=\frac{A\left(\mathbf{q}_{1,2}, \omega_{m_{1,2}}\right),\left(C\left(\mathbf{q}_{1,2}, \omega_{m_{1,2}}\right), B\left(\mathbf{q}_{1,2}, \omega_{m_{1,2}}\right)\right)}{\mathbf{q}^{2} A^{2}\left(\mathbf{q}_{1,2}, \omega_{m_{1,2}}\right)+\omega_{m_{1,2}}^{2} C^{2}\left(\mathbf{q}_{1,2}, \omega_{m_{1,2}}\right)+B^{2}\left(\mathbf{q}_{1,2}, \omega_{m_{1,2}}\right)} .
$$

\subsection{Angular integration}

As seen in Eqs. (8)-(11) the tBS equation implicitly depends on three spatial solid angles, $\left(\theta_{\mathbf{p}}, \varphi_{\mathbf{p}}\right),\left(\theta_{\mathbf{q}}, \varphi_{\mathbf{q}}\right)$ and $\left(\theta_{\mathbf{p}}, \varphi_{\mathbf{p}}\right)$. The dependence on $\left(\theta_{\mathbf{q}}, \varphi_{\mathbf{q}}\right)$ and $\left(\theta_{\mathbf{p}}, \varphi_{\mathbf{p}}\right)$ follows from the interaction kernel $D\left(\kappa^{2}=(\mathbf{p}-\mathbf{q})^{2}+\left(\omega_{m}-\omega_{n}\right)^{2}\right)$, which consists of two parts, $D_{1}\left(\kappa^{2}\right) \sim \mathrm{e}^{-\kappa^{2} / \omega^{2}}$ and $\left.D_{2}=\kappa^{2} / \omega^{2} D_{1}\left(\kappa^{2}\right)\right) \equiv \alpha \kappa^{2} \exp \left(-\alpha \kappa^{2}\right)$. The dependence on $\left(\theta_{\mathbf{P}}, \varphi_{\mathbf{P}}\right)$ and also on $\left(\theta_{\mathbf{q}}, \varphi_{\mathbf{q}}\right)$ comes from the propagator functions $\sigma_{F_{1}}(1)$ and $\sigma_{F_{2}}(2)$. The angular parts of $D_{1}$ and $D_{2}$ of the kernel can be handled by decomposing them over the spherical harmonics $\mathrm{Y}_{l m}(\mathbf{q})$ and $\mathrm{Y}^{*}{ }_{l m}(\mathbf{p})$ as

$$
D_{1}\left(\kappa^{2}\right) \equiv \mathrm{e}^{-\kappa^{2} / \omega^{2}}=\mathrm{e}^{-\left[\left(\omega_{n}-\omega_{m}\right)^{2}+(|\mathbf{q}|-|\mathbf{p}|)^{2}\right] / \omega^{2}} 4 \pi \sum_{l m} f_{l}^{(s)}\left(2|\mathbf{q} \| \mathbf{p}| / \omega^{2}\right) \mathrm{Y}_{l m}(\mathbf{q}) \mathrm{Y}^{*}{ }_{l m}(\mathbf{p}),
$$


where the coefficients $f_{l}^{(s)}\left(2|\mathbf{q}||\mathbf{p}| / \omega^{2}\right)$ (here $\omega^{2}$ denotes the slope parameter of the AWW kernel) are proportional to the scaled spherical Bessel functions of the first kind, $I_{n}(x)$. The second part of the kernel can be found by observing that $D_{2}\left(\alpha \kappa^{2}\right)=-\alpha \frac{d D_{1}\left(\alpha \kappa^{2}\right)}{d \alpha}$, where $\alpha=$ $1 / \omega^{2}$.

The angular dependence on $\left(\theta_{\mathbf{P}}, \varphi_{\mathbf{P}}\right)$ and $\left(\theta_{\mathbf{q}}, \varphi_{\mathbf{q}}\right)$, which comes from the propagator functions, is calculated in an analogous manner as in Eq. (12)

$$
\sigma_{F_{1}}\left(p_{1}\right) \sigma_{F_{2}}\left(p_{2}\right)=\sum_{L} \sigma_{L}^{F_{1} F_{2}}\left(\omega_{n}, \Omega_{N},|\mathbf{q}|,|\mathbf{P}|\right) \sum_{M} \mathrm{Y}_{L M}(\mathbf{P}) \mathrm{Y}_{L M}^{*}(\mathbf{q})
$$

In the expressions above, the superscripts $F_{1} F_{2}$ denote the decomposition of diagonal $F_{1} F_{2}=V V, S S, C C$ and non-diagonal products $V S, S V, V C, C V, C S, S C$ of the propagator functions, respectively.

\section{Results}

We solve the tBS equation for pseudo-scalar ground states for two values of the Matsubara frequency $N=1$ and $N=2$. At given temperature and Matsubara frequency the possible value of the pole mass is restricted to the interval $4 \pi^{2} N^{2} T^{2}>M_{q \bar{q}}^{2} \geq 0$ which corresponds to $0<|\mathbf{P}|^{2}<4 \pi^{2} N^{2} T^{2}$. The maximum value of the pole mass corresponds to the limit of thermal mass, i.e. $|\mathbf{P}| \rightarrow 0$. For the lowest Matsubara frequency, $N=1$, this limit can occur already at $T \sim 100 \mathrm{MeV}$, which implies that the solution of tBS at large temperatures approaches the thermal limit $|\mathbf{P}| \rightarrow 0$. Results of calculations for the ground state pseudo-scalar pole masses are presented in Fig. 1 for the Matsubara frequencies $N=1$ (solid curve) and $N=2$ (dashed curve) as functions of temperature. In both cases, the mass rapidly increases with increasing temperature, and already at $T \geq 100 \mathrm{MeV}$ (for $N=1$ ) and $T \geq 80 \mathrm{MeV}$ (for $N=2$ ) becomes larger than the maximum value of the mass of two quasi-free quarks at the same value of $T$. This is demonstrated in Fig. 1, where the mass of two quarks, defined as the solution of tDS equation, $M_{2 q}=2 B\left(\mathbf{0}, \omega_{0}\right) / A\left(\mathbf{0}, \omega_{0}\right)$, is presented by the dotted curve.

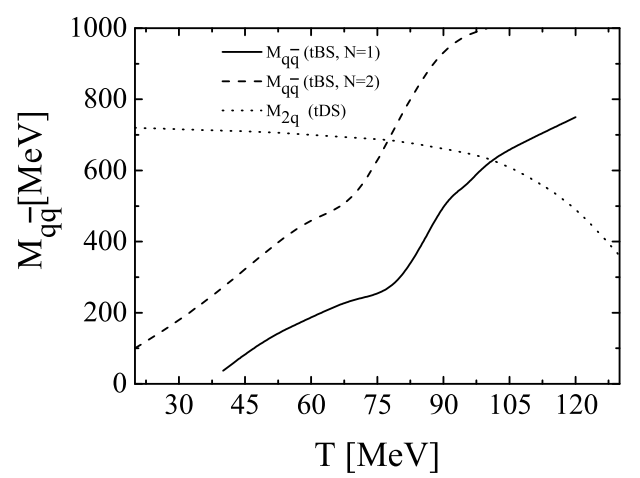

Figure 1. The solution of the tBS equation with the AWW kernel for pseudo-scalar mesons for the Matsubara frequency $N=1$, solid curve and $N=2$, dashed curve. The dotted line represents the maximum value of the two quasi-free quark masses as the solution of the tDS equation for the Matsubara frequency with $n=0$ and zero three momentum $|\mathbf{k}|=0$

For temperatures, where $M_{q \bar{q}}>M_{2 q}$, the bound ground state, in the "canonical" sense, does not exists or can be considered as unstable against the dissociation into two correlated quasi-free quarks.

\section{Summary}

We have investigated the solution of the truncated Dyson-Schwinger (tDS) equation at finite temperature within the rainbow approximation by employing the Alkofer-Watson-Weigel 
(AWW) model which consists only of the infrared term in a more complex interaction kernel. The solution of the tDS equation is a prerequisite for a consistent solution of the truncated Bethe-Salpeter (tBS) equation for quark-antiquark bound states at finite temperature within the same approximation. We investigate to what extent the model, which provides a fairly good description of ground-state mesons at zero temperatures, can be applied to the truncated tDS equation at finite temperatures.

In our calculations, we restrict the boson Matsubara frequency $\Omega_{N}=2 \pi N T$ to $N=1$ and $N=2$. Larger values of $N$ provide large values of $M_{q \bar{q}}(N>2)$, far above masses of known lightest pseudo-scalar mesons. For each Matsubara frequency $\Omega_{N}$, the ground state mass $M_{q \bar{q}}^{2}=4 \pi^{2} N^{2} T^{2}-\mathbf{P}^{2}$ is defined as the first (lowest) zero of the corresponding determinant as a function of $|\mathbf{P}|$. Such a mass is referred to as the Matsubara pole mass.

For both frequencies $\Omega_{N}, N=1$ and $N=2$, we find that the pole masses rapidly increase with increase of temperature. This is in agreement with the behaviour of the screening masses at large temperatures reported in literature, see e.g. [22].

At large values of $T, T>100 \mathrm{MeV}$, the pole masses become larger than the sum of two quarks. This implies that at large $T$ the ground state of two quark does not occur in the sense as commonly adopted in quantum mechanics where the binding energy is negative. This can be interpreted as dissociation instability of the state against a fragmentation into a state of two quasi-free quarks.

\section{References}

[1] C.S. Fischer, S. Kubrak, R. Williams, Eur. Phys. J. A 51, 10 (2015)

[2] T. Hilger, M. Gómez-Rocha, A. Krassnigg, Eur. Phys. J. C 77, 625 (2017)

[3] P. Maris, C.D. Roberts, Phys. Rev. C 56, 3369 (1997)

[4] P. Maris, P.C. Tandy, Phys. Rev. C 60, 055214 (1999)

[5] S.M. Dorkin, L.P. Kaptari, B.Kämpfer, Phys. Rev. C 91, 055201 (2015)

[6] T. Hilger, C. Popovici, M. Gomez-Rocha, A. Krassnigg, Phys. Rev. D 91, 034013 (2015)

[7] P. Maris, C.D. Roberts, Int. J. Mod. Phys. E 12, 297 (2003)

[8] A. Krassnigg, P. Maris, J. Phys. Conf. Ser. 9, 153 (2005)

[9] S.M. Dorkin, T. Hilger, L.P. Kaptari, B. Kämpfer, Few Body Syst. 49, 247 (2011);

S.M. Dorkin, L.P. Kaptari, C. Ciofi degli Atti, B. Kämpfer, Few Body Syst. 49, 233 (2011)

[10] S. -x. Qin, L. Chang, Y. -x. Liu, C.D. Roberts, D.J. Wilson, Phys. Rev. C 84, 042202 (2011); ibid. Phys. Rev. C 85, 035202 (2012)

[11] M.R. Frank, C.D. Roberts, Phys. Rev. C 53, 390 (1996)

[12] R. Alkofer, P. Watson, H. Weigel, Phys. Rev. D 65, 094026 (2002)

[13] C.S. Fischer, P. Watson, W. Cassing, Phys. Rev. D 72, 094025 (2005)

[14] R. Alkofer, L. von Smekal, Phys. Rept. 353, 281 (2001)

[15] T. Matsubara, Prog. Theor. Phys. 14, 351 (1955)

[16] J.I. Kapusta, Finite-Temperature Field Theory (Cambridge University Press, N.Y., 1989)

[17] A.A. Abrikosov, L.P. Gorkov, I.E. Dzyaloshinski, Methods of Quantum Field Theory in Statistical Physics (PRENTICE-HALL, INC., Englewood Cliffs, New Jersey, 1963)

[18] N. Souchlas, arXiv:nucl-th/1006.0942

[19] C.S. Fischer, J.A. Mueller, Phys. Rev. D 80, 074029 (2009)

[20] S.M.Dorkin, M. Viebach, L.P. Kaptari, B. Kämpfer, J. Mod.Phys. 7, 2071 (2016)

[21] S. Llewellyn, Ann. of Phys. 53, 521 (1969)

[22] M. Ishii, H. Kouno, M. Yahiro, Phys. Rev. D 95, 114022 (2017) 